\title{
Über die Korrektion von leichter Myopie durch neue Behandlung mit Gummidruckverband.
}

\author{
Von
}

\author{
Dr. Tatsuji Inouye, \\ Surugadai, Tokyo, Japan. \\ Mit 7 Textabbildungen.
}

Wenn man mit beginnender Myopie zu tun hat, ist es wünschenswert, daß es irgendeine Methode gibt, ohne Konkavgläser die normale Sehschärfe wieder herzustellen. Mein Gummidruckverband ist bei einem solchen Falle zu probieren.

Als ich Fälle von Staphyloma corneae durch Gummidruckverbände zu beeinflussen suchte (s. vorstehende Arbeit), konnte ich manch-

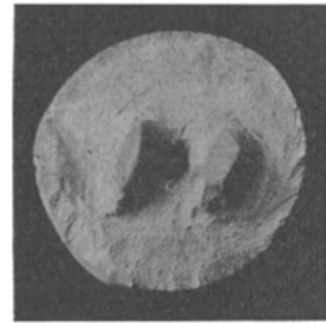

Abb. 13. Gummiform zur Behandlung des Astigmatismus von Fall $I$, Innenansicht. (1/2 natiriliehe Größe.)

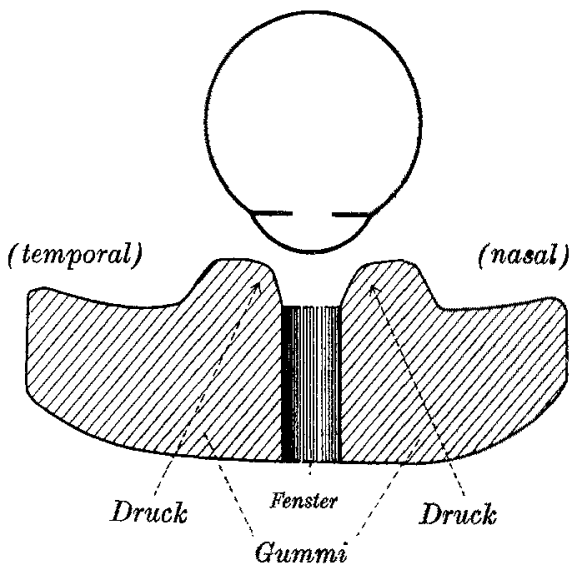

Abb. 14. Durchschnit dureh zwel Vorstalpungen von $A b b .13$ und durch das Auge. Das Fenster ist zur Kontrolle, dab diese stelle genau uber der Mitte der Hornhaut liegt. (Natürliche Gröbe)

mal eine abnorme Abflachung der Cornea als Folge des Verbandes an der Stelle des Staphyloms makroskopisch nachweisen. Die Abflachung der Cornea bedeutet eine Vergrößerung des Krümmungsradius der Hornhaut. Die Vergrößerung um $1 \mathrm{~mm}$ genügt, um eine Myopie von annähernd sechs Dioptrien zur Emmetropie zu korrigieren. Diese Abflachung der Cornea war mir der Beweggrund, Versuche zu machen, 


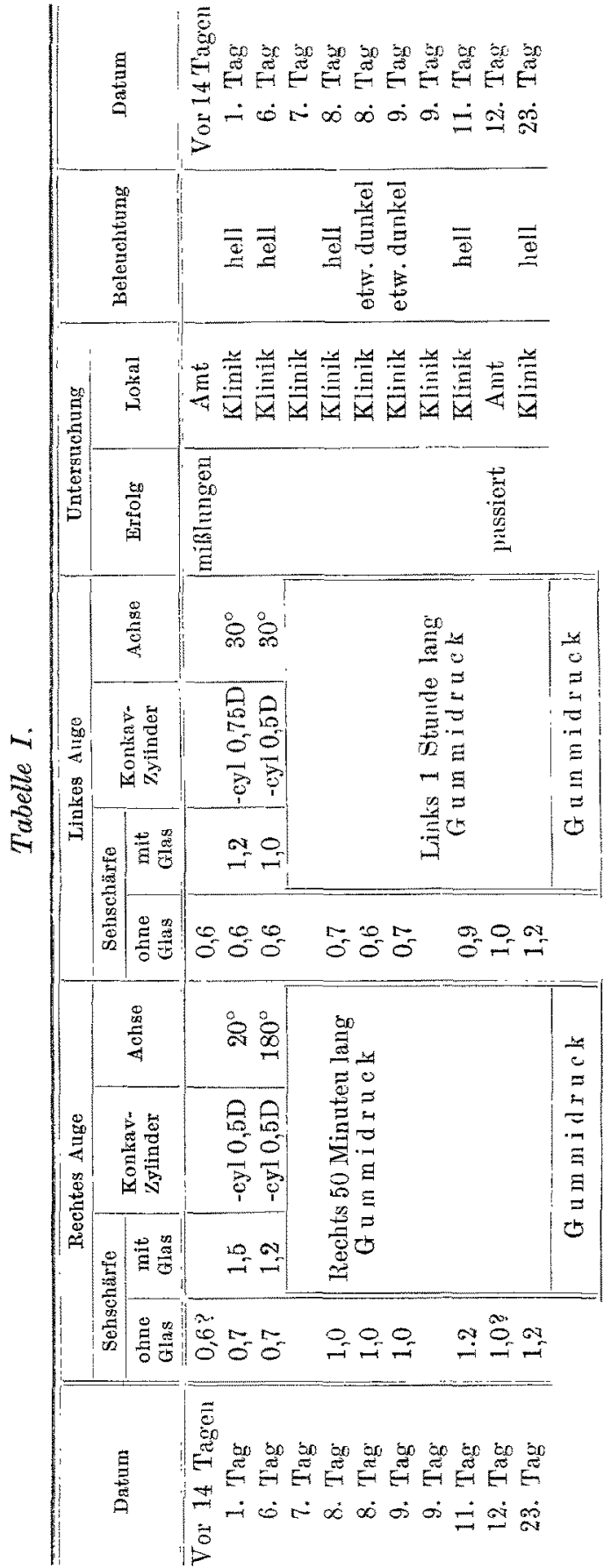

nicht nur das Staphylom, sondern auch die Myopie durch Gummikompression zu korrigieren.

Wenn die Augenachse um $1 \mathrm{~mm}$ verkürzt wird, so ist eine Myopie von nähernd drei Dioptrien zur Emmetropie korrigiert. Diese Tatsache war schon lange im allgemeinen nachgewiesen. Die Augenachse soll bei der Kompression der Cornea verkürzt, wenigstens aber nicht verlängert werden.

Im Jahre 1912 habe ich eine besondere Art von Astigmatismus beobachtet. Tumoren hatten von der Seite auf die Bulbi gedrückt; so war beim ersten Falle $3,5 \mathrm{D}$, beim zweiten Falle 2,5D regelmäßiger Astigmatismus zu erkennen. (Näheres siehe im Literaturnachweis). Deshalb habe ich zuerst Astigmatismus myopicus (Fall 1) gewählt. Um diese Astigmatismen zu driicken, hatte ich besondere Gummitormen gebildet mit zwei Vorstiilpungen. (Abb. 13 und 14.) Diese Vorstülpungen haben den Limbus an zwei Stellen zu drücken. 
Diese zwei Stellen entsprechen gerade dem Meridian mit schwächster Hornhautkrümmung.

Die Achsen der Hornhaut sind hier nach internationaler Bezeichnung angeführt. (Über mein besonderes System der Achsenbezeichnung vergleiche die Literaturangabe.) Die Sehschärfe wird bei allen Fällen nur mit Landoltschem Ring gemessen, weil unser Marineamt diesen Ring gebraucht. Ich messe sonst gewöhnlich nur mit einfachen Haken von $5 \mathrm{~mm}$ Durchmesser für 1,0 Sehschärfe, die schneller und sicherer die Sehprobe gestatten. (Siehe Literaturangabe.)

Fall 1. Take. Yana. Seeoffizier der Handelsmarine.

1. Tag, 27. II. 1917. Mit Probierglas fand ich Astigmatismus myopicus. R. V. $=0,7$ ohne Glas; aber r. V. $=1,5$ mit Konkavzylinder $-0,5$ D, dessen Achse $20^{\circ} ; 1 . \mathrm{V} .=0,6 ; 1,2 \mathrm{~m}-0,75 \mathrm{D}$, Achse $30^{\circ}$, siehe Tabelle I, ophthalmoskopisch Hornhautastigmatismus, siehe Tabelle II. Wann diese Sehstörung entstand, ist nicht klar, aber der Patient merkte dieselbe zuerst bei der marineamtlichen Untersuchung, 14 Tage vor meiner ersten Konsultation.

Am 7. Tage wurde das rechte Auge direkt vor dem Sohlafengehen 50 Minuten lang gedrückt. (Gummiform siehe $A b b .13$.) Beim Druck sa B Patient mit geöffnetem linken Auge in richtiger Haltung vor einem großen Spiegel. Die Sehschärfe stieg von 0,7-1,0 am nächsten Tage durch diesen einmaligen Druck, und später sogar bis 1,2. Die Sehsehärfe des linken Auges, welches keinen Druckverband an demselben Tage bekam, blieb fast in gleiohem Zustand, siehe Tabelle $I$.

9. Tag. Das linke Ange wurde gedrückt, die Sehschärfe stieg von $0,7-0,9$ und später sogar bis 1,2 .

12. Tag. Er hat die Untersuchung in dem Seeamt anstandslos passiert für die beste Klasse.

23. Tag. Keine Wiederverschlechterung der Sehschärfe, sogar weitere subjektive und objektive Besserung.

Nach einem Jahre habe ich von einem Kollegen gehört, daß die Sehschärfe noch in gutem Zustand geblieben sei. Bei diesem Falle gewann ich den ersten Anhaltspunkt, daß Gummidruck einen bedeutenden Einfluß auf die Besserung der Sehschärfe bei Astigmatismus myopicus haben kann.

Fall 2. Ken. Ima. Dieser Fall ist in Tabelle II nachzusuchen. Es wurde im Frühling 1917 gedrückt. Die Gummiform wax wie bei Fall 1. Der Druck verursachte aber Augenschmerz. Patient wurde ambulant behandelt, also nicht aufgenommen. So konnte ich ihn nicht direkt vor Schlafengehen drücken und auch kein befriedigendes Resultat erhalten. Die Sehschärfe ist trotzdem mehr gestiegen als bei Fall 3, welcher Fall amtliche Untersuchungen glücklich passierte.

Fall 3. Tok. Sho. wurde im Mai 1918 gedrückt. Die rechte Sehschärfe war bloß 0,3 direkt vor dem Examen, doch passierte er trotzdem.

Die Regel des Seeamtes lautet: Wenn die Sehschärfe 0,4 auf dem schlechteren Auge beträgt, aber auf dem besseren Auge mindestens 0,6 , so ist Passierung in die 2.Klasse möglich (die ich in Tabelle II als Pass + bezeichnet habe). Zur Passierung in 1. Klasse wird eine noch bessere Sehschärfe verlangt, die ich als Pass ++ bezeichnet habe, wie bei Fall 1.

Die Gummiformen von Fall 3-16 sind anders als won Fall $1-2$ und bilden stath 2 Vorstülpungen eine einfache konkave Fläche gegen die Cornea. Mit diesen neuen Gummiformen fühlten die Patienten weniger Augenschmerzen. (Siehe auch Abb. 15, $16,19$. 
Fall 4. To. Ya, 20 Jahre, Schüler der Seemannsschule. Im August 1918 wurde das rechte Auge gedrückt, während das linke nicht verbundene Auge in den Spiegel gesehen hatte, der Schüler schlief gleich danach ein.

Fall s. Sei. Su.

1. Tag war 2. III. 1919. Er wurde von einem Augenarzt meiner Behandlung empfohlen. Seine Sehsehärfe war nach Angaben des Kollegen $r . \mathrm{V} .=0,4 ; 1,0$ mit $-1,0 \mathrm{D} ; \mathrm{l}$. V. $=1,0$. Meine Sehschärfeprüfung zeigte $\mathrm{r} . \mathrm{V} .=0,7$, partiell $=0,7(\mathrm{p})$; $1,2 \mathrm{~m}-1,0 \mathrm{D} ; \mathrm{l} . \mathrm{V} .=0,9(\mathrm{p}) ; 1,2 \mathrm{~m}-0,5 \mathrm{D}$.

5. Tag. Vormittag $\mathrm{r} . \mathrm{V} .=0,9 ; 1,2(\mathrm{p}) \mathrm{m}-0,25 \mathrm{D} ; 1,2 \mathrm{~m}-0,5 \mathrm{D} ; \mathrm{l} . \mathrm{V} .=1,2$. Gegen 8 Uhr Abends wurde rechts mit Gummiform (Tag-Gummiform) gedrutokt und vor einem $3 \mathrm{~m}$ entfernten Spiegel gesessen, wäbrend er seinen Schatten fixierte. Weil er möglichst wenig blinzeln sollte, hatte ex 12 Minuten nach dem Druckverband heftige Augenschmerzen, Sohweißsekretion und Brechneigung; 17 Minuten lang wurde der Verband fortgesetzt, dann weggenommen, danach konnte er sofort einschlafen,

6. Tag. R. $V_{n}=0,8 ; 1,2(p) m-0,5 \mathrm{D} ; 1 . \mathrm{V}_{0}=1,2(\mathrm{p})$. In derselben Nacht, direkt vor dem Schlafengehen, wurde das rechte Oberlid, anstatt mit der Gummiform, mit seinem eigenen Mittelfinger sanft und kontinuierlich geschlossen und dabei auf das Lid unterhalb des oberen Orbitalrandes 40 Minuten lang gedruckt. Keine Schmerzen, aber auch keine nennenswerte Wirkung.

7. Tag. R. V. $=0,8 ; 1,2 \mathrm{~m}-0,25 \mathrm{D} ; 1, \mathrm{~V} .=1,2$. In der Nacht wurde mit neuer Gummiform (Nacht-Gummiform) über seinem rechten geschlossenen Lid gedrückt, gleich eingeschlafen. Nach 1 Stunde wurde dieser Verband weggenommen, und gleich wieder eingeschlafen. Nacht-Gummiform drückte die Cornea mehr von oben vorn als Tag-Gummiform, weil beim geschlossenen Lid die Cornea höher steht. Nacht-Gummiform war angenehmer als Tag-Gummiform für ihn gewesen.

8. Tag. R. V. $=0,9 ; 1,2 \mathrm{~m}-0,25 \mathrm{D} ; 1$. V. $=1,2$. Am 10. III. hat er die Untersuchung ++ passiert. Bei diesem Falle ist besonders zu bemerken, daß die Sehschärfe vom 1. bis 5. Tage von 0,7-0,9 gestiegen ist. Dieser Umstand scheint mir nicht nur durch den Akkommodationskrampf erklärlich, sondern auch zum Teil durch den Druck, welcher durch wiederholtes Ansetzen der Gummiform beim Probieren vor der Anwendung unvermeidlich war.

Fall 6. Toshi. Nishi, kam im März 1919 zur ersten Untersuchung, Am 18. V. erster Druck links. Vorn 19. VI. bis 13. X. Einstellen des Druckes, weil der Sommer keine gute Jahreszeit ist, wm solche relativ hochgradige Myopie zu drücken. Die Gummiformen (Nacht-Gummiform) sind alle auf geschlossenen Augen zu brauchen.

Fall 7. Ji. Ko. kam am 25. III. 1919 zu meiner ersten Untersuchung mit erweiterten Pupillen. Er wurde seit 40 Tagen von einem Augenarzt behandelt, um vom Akkommodationskrampf befreit zu werden. Er glaubt keinen Erfolg gehabt zu baben. Er blinzelt sehr häufig. Er wurde im April und Mai 12 mal gedrückt, zum Teil auf gesehlossenen Augen mit einer Nacht-Gummiform, zum Teil auf geöffneten Augen mit einer Tag-Gummiform.

Fall 8. Ma. Ogu. wurde im Mai und Juni 1919 gedrückt. Leichte Bindehaut: entzündung, wurde mit Alaun und so weiter behandelt. Diese Gummiform war bei geöffnetem Zustande des anderen Auges zu brauchen.

Fall 9. Ryo. Fuji. wurde im Oktober 1919 mit gutem Erfolg gedrückt. Links wurde $2 \mathrm{mal}$ gedrückt. Nach erstmaligem Druck zeigte sich skiaskopisch rechts im schwachgekriimmten. Meridian - 0,25 D, im starkgekrümmten Meridian - $1,5 \mathrm{D}$ : Achse ungefähr in $100^{\circ}$. Links zeigte sich im ersten Meridian $-1,5 \mathrm{D}$, im zweiten $-2,5 \mathrm{D}$; Achse $100^{\circ}$. Nach vollendeter Druckbehandlung bestand rechts im ersten - 1,0 D, im zweiten - 2,0 D; Achse 105. Er akkommodierte dabei besonders heftig. Links bestand dabei im ersten $-1,0 \mathrm{D}$, im zweiten -2,0 D; Achse $100^{\circ}$. 
Es fand sich skiaskopisch durchschnittlich $\mathrm{r},-1,25 \mathrm{D} ; \mathrm{l},-1,75 \mathrm{D}$, wie in Tabelle II steht. Leichtes papilläres Trachom, wurde mit Argentum nitricum und so weiter behandelt.

Fall 10. Kon. Miya. wurde zuerst im Dezember 1919 gedrückt. Diese Gummiform war auf geschlossenen Augen zu brauchen.

Fall 11. Gon. Naka. kam im Dezember 1919 wegen der Myopie zu mir. Im Oktober $1920 \mathrm{kam}$ er wieder und wurde gedrückt. Wegen der akuten Conjunctivitis wurde er bloß 2 mal gedrüekt.

Fall 12. Toku. Uye., 31 Jahre, Seeoffizier der Handelsmarine.

1. Tag war 14. I. 1920. Er erinnert sich, daß er in seinem 20. Jahre schon im Theater die Bühne nicht deutlich sehen konnte. Vor 4 Tagen Untersuchung vom Amt, Annahme abgelehnt. Er hat mir eine genau geschriebene Krankengeschichte freundlicher Weise gegeben, so kann ich hier seine subjektiven Gefühle

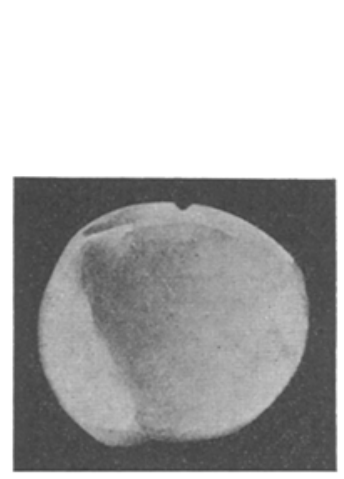

Abb. 15. Gummiform zur Behandinng der Myopie von Fall 12. Sogenannte NachtGuxmmiform. Innenansicht. (1/2 natürliche GröBe.)

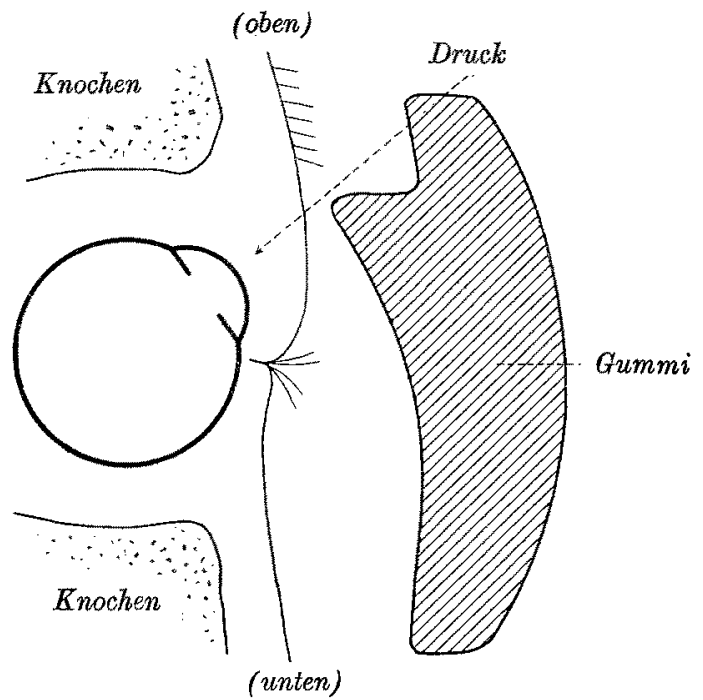

Abb. 16. Sagittaler Durchschnitt durch das geschlossene Auge yon Fall 12. (Natürliche Gröbe.)

bei dieser Behandlung angeben. R. V. $=0,5(\mathrm{p}) ; 0,8(\mathrm{p}) \mathrm{m}-0,5 \mathrm{D} ; 1,2 \mathrm{~m}-0,75 \mathrm{D}$; 1. $V .=0,3 ; 0,6(\mathrm{p}) \mathrm{m}-0,5 \mathrm{D} ; 1,2(\mathrm{p}) \mathrm{m}-0,75 \mathrm{D}$. Leichte chronische Conjunctivitis auf beiden Augen.

5. Tag. Gipsabguß von beiden Augen genommen.

6. Tag. In die Klinik aufgenommen. Skiaskopie in $50 \mathrm{~cm}$ Entfernung zeigte r. 1. $-1,25 \mathrm{D}$.

7. Tag. Vormittag r. V. $=0,4 ; 1,2 \mathrm{~m}-1,0 \mathrm{D} ; 1 . \mathrm{V} .=0,5$. Die rechte halbgebildete Gummiform zuerst auf Gipsform gesetzt und umgeformt und dann auf seinem Auge probiert, Vormittags und Nachmittags häufig angesetzt und umgeformt. Gegen 6,30 Uhr fertig. 7,30 Uhr abends rechts Druckverband. Mit Verband lag er sofort auf dem Rücken im Bette. Beide Augen sollen geschlossen sein. Die Gummiform war also Nacht-Gummiform. Seitenlage ist verboten. Einschlafen war natürlich erlaubt, doch schlief er nur kurze Zeit. Wegen Augensehmerz wachte er auf, 8,40 Uhr, Verband sich selbst nach meiner Ordination weggenommen. Nach Wegnahme sah er allmählich alle Gegenstände deutlicher. Als er ganz deutlich zu sehen anfing, ging Augenschmerz vollständig weg; gleich eingeschlafen. 
8. Tag. Subjektiv deutlicher und die Sehschärie $\mathrm{r} . \mathrm{V} .=0,6 ; 1,2 \mathrm{~m}-0,75 \mathrm{D}$; l, V. $=0,4 ; 1,2 \mathrm{~m}-1,0 \mathrm{D}$. 1 mal Spülung der Augen, 2 mal kalte Umschläge, 3 mal verdünnte 0,25 proz. Cocainlösung und 2 mal 2 proz. Argyrollösungen eingeträufelt. Diese Behandlung gegen Conjunctivitis wurde fast an jedem Tage fortgesetzt. Rechts 80 Minuten lang wie voriges Mal gedrückt, kein Angenschmerz.

12. Tag. Mit etwas modifizierter Nacht-Gummitorm. (Abb. 15 and 16.) Rechts 80 Minuten lang gedrückt wie roriges Mal; der Verband war bei der Abnahme bereits etwas zu locker geworden. Kein Augenschmerz.

19. Tag. R. V. $=0,6 ; 1,2 \mathrm{~m}-0,75 \mathrm{D} ; 1 . \mathrm{V} .=0,6(\mathrm{p}) ; 1,2 \mathrm{~m}-0,75 \mathrm{D}$. Nachts 80 Minuten lang Druckverband. Verband war gut angepaßt, gleich eingeschlafen.

20. Tag. Letzte Nacht gut geschlafen. R. V. $=0,8 ; 1,2(\mathrm{p})-0,5 \mathrm{D} ; 1 . \mathrm{V}$. $=0,6 ; 1,2 \mathrm{~m}-0,5 \mathrm{D}$. Er fühlt deutlich, då die rechte Sehschärfe seit 3 oder 4 Jahren nie so gut gewesen ist wie jetzt.

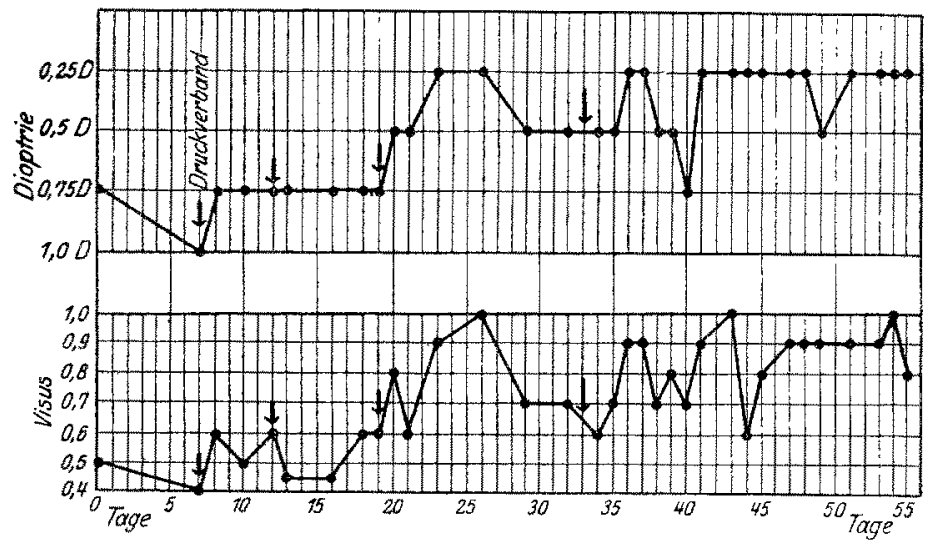

Abb. 17. Die Veränderung der Sehsehärfe und der Dioptriezahl der Konkavgläser während der Druckbehandluag. Fall 12 , rechtes Ange. Nach viermaligem Druek wurde die Dioptriezahl von $-0,75 \mathrm{D}$ bis $-0,25 \mathrm{D}$ vermindert und die Sebschärie von 0,5 bis 0,9 gebessert.

26. Tag. R. V. $=1,0 ; 1,2 \mathrm{~m}-0,25 \mathrm{D} ; 1 . \mathrm{V} .=0,6 ; 1,2 \mathrm{~m}-0,5 \mathrm{D}$. Er merkt, daß die Buchstaben mit dem rechten Auge auf der Straße alle sehr deutlich zu sehen sind, während sie mit dem noch nicht behandelten linken Auge nicht deutlich zu sehen waren.

32. Tag. R. V. $=0,7(p) ; 1,2(p) \mathrm{m}-0,5 \mathrm{D} ; 1 . \mathrm{V} .=0,6(\mathrm{p}) ; 1,2(\mathrm{p}) \mathrm{m}-0,75 \mathrm{D}$.

33. Tag. Abends Druckverband 1 Stunde lang, dabei war der Verband zu fest, schmerzhaft, Brechneigung; $8,30 \mathrm{Chr}$ sanft eingeschlafen.

35. Tag. R. V. $=0,7 ; 1,2 \mathrm{~m}-0,5 \mathrm{D} ; 1 . \mathrm{V} .=0,6 ; 1,2 \mathrm{~m}-0,5 \mathrm{D}$. Links Druckverband.

40. Tag. Links Druckverband.

47. Tag. Skiaskopie in $50 \mathrm{~cm}$ Entfernung zeigte $\mathrm{x}$. und 1. -1,0 D.

49. Tag, Ich habe gemerkt, daß die linke Cornea bei geschlossenem Lide sich nicht gerade nach oben richtete, sondern $6 \mathrm{~mm}$ nach oben und $7 \mathrm{~mm}$ nach außen, deswegen habe ich die linke Gummiform etwas geändert. R. V. $=0,9$; 1,2 (p) $\mathrm{m}-0,5 \mathrm{D} ; \mathrm{l}$. V. $=0,6 ; 1,2$ (p) $\mathrm{m}-0,5 \mathrm{D}$.

50. und 53. Tag. Links Druckverband.

54. Tag. R. V. $=1,0 ; 1,2 \mathrm{~m}-0,25 \mathrm{D} ; \mathrm{l} . \mathrm{V} .=0,9(\mathrm{p}) ; 1,2(\mathrm{p}) \mathrm{m}-0,25 \mathrm{D}$. Die Besserung der Sehschärfe während unserer Behandlung ist auf $\mathrm{Abb}, 17$ ersichtlich.

56. Tag. Passierung der Untersuchung vom Amt mit bestem Erfolg. 
Fall 13. Shin. Ama kam am 10. III. 1920 za mir. Eine auf dex anderen Straßenseite angeschriebene Telephonnummer konnte er nach der Behandlung leichter lesen. Vor der Druckbehandlung bestand wegen des Astigmatismus, sowohl

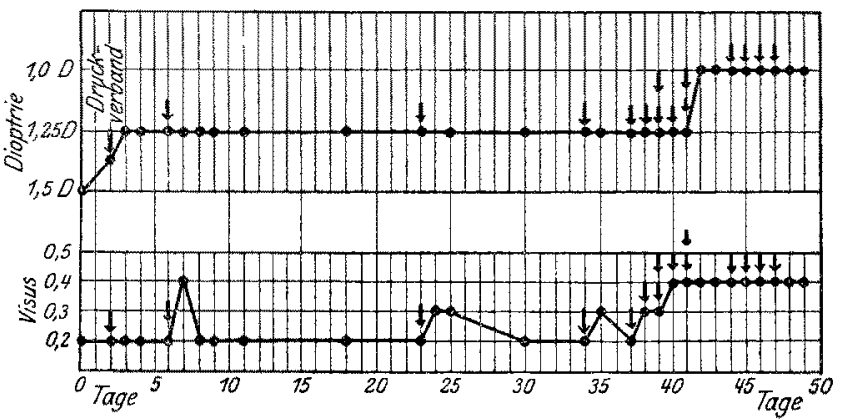

Abb. 18. Die Veränderung der Sehschärfe und der Dioptrienzah1. Fall 15, rechtes Auge.

auf dem rechten wie auf dem linken Auge, ohne Glas monokuläres Doppelsehen. Nach der Behandlung sah er die Buchstaben einfach.

Fall 14. Ko. Miya wurde zuerst im März 1920 gedrückt. Vor Druck r. V. $=0,5$; Konkavgläser steigerten die Sehschärfe nicht. Nach Druck r. $\mathrm{V} .=0,6 ; 1,0 \mathrm{mit}-0,75 \mathrm{D}$ geworden.

Fall 15. (Dazu Abb. 18.) Tora Take, 27 Jahre. Die Abb. 18 gibt einen Überblick über diesen Fall. Das rechte Auge wurde zuerst 4 mal gedrückt, und 3 mal hatte sich der Visus unmittelbar danach gebessert, war aber glejch wieder auf den früheren Zustand zurückgegangen. So drückte ich täglich 1-2 mal (Tag und Nacht) jedesmal $1-3$ Stunden lang. Durch diese forcierte Druckanwendung hatte sich die Sehschärfe von 0,2-0,4 gebessert. Es wurden zwei Arten Gummiformen angewendet, eine bei geöffnetem, die andere bei geschlossenem $\mathrm{Zu}$ -

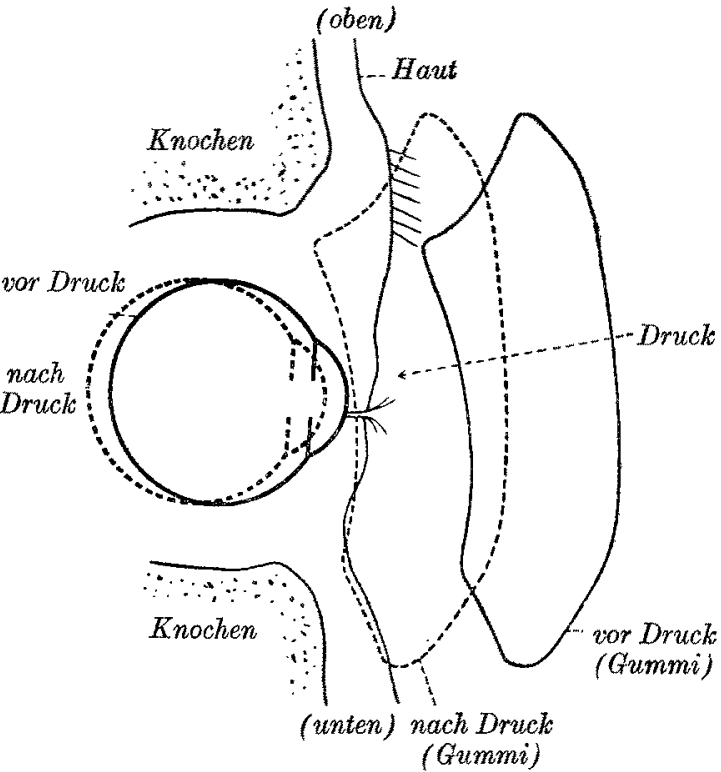

Abb. 19. Sagittaler Durohschnitt durch das rechte Auge. Sogenannte Taggummiform von Fall 15. Das reohte Auge ist gerade tach vorn gerichtet, vom Lid vollstandig bedeckt. Durch den Druck des Gummis wird das Auge nach rỉekwärts bis zur punk: tierten Lage verseho ben. (Natürliohe GröBe.) stande des anderen Auges.

Diese heißt Nacht-Gummiform und jene Tag-Gummiform (Abb. 19).

Fall 16. Ku. Kashi wurde im Juni 1921 gedrückt. Näheres siehe Tabelle II. Der Fall bot der Behandlung besondere Schwierigkeiten, da erst das Trachom gebessert werden mußte. 
Tabelle II.

\begin{tabular}{|c|c|c|c|c|c|c|c|c|c|c|}
\hline \multirow{3}{*}{$\begin{array}{l}\text { Fali. } \\
\text { Nr. }\end{array}$} & \multirow{3}{*}{ Name } & \multirow{3}{*}{ Alter } & \multirow{3}{*}{ Beruf } & \multirow{3}{*}{$\begin{array}{c}\text { Rechts } \\
\quad R \\
\text { Links } \\
I\end{array}$} & \multirow{3}{*}{$\begin{array}{c}\text { Dauer der } \\
\text { Myopie } \\
\text { oder des } \\
\text { Astigm. }\end{array}$} & \multicolumn{4}{|c|}{ Javalsches Ophthalmometer } & \multirow{3}{*}{ Skiaskop } \\
\hline & & & & & & \multicolumn{2}{|c|}{$\begin{array}{l}\text { sichwächster Halb- } \\
\text { durchmesser der } \\
\text { Correat und Achse }\end{array}$} & \multicolumn{2}{|c|}{$\begin{array}{l}\text { Differenz zwischen } \\
\text { schwächstem und } \\
\text { stärkstem Meridian }\end{array}$} & \\
\hline & & & & & & $\begin{array}{c}\text { vor } \\
\text { Druek }\end{array}$ & $\begin{array}{l}\text { nach } \\
\text { Druck }\end{array}$ & $\begin{array}{c}\text { vor } \\
\text { Druck }\end{array}$ & $\begin{array}{l}\text { nach } \\
\text { Druek }\end{array}$ & \\
\hline \multirow[t]{2}{*}{1} & $\begin{array}{l}\text { Take. } \\
\text { Yana }\end{array}$ & 27 & $\begin{array}{l}\text { See- } \\
\quad \text { offizier }\end{array}$ & $\mathbf{R}$ & $\begin{array}{c}\text { uber } \\
15 \text { Tage }\end{array}$ & $\mid \begin{array}{c}8,17 \mathrm{~mm} \\
180^{\circ}\end{array}$ & & $-0,5 \mathrm{D}$ & & \\
\hline & & & & $\mathrm{L}$ & $\begin{array}{c}\text { über } \\
15 \text { Tage }\end{array}$ & $\begin{array}{c}8,22 \mathrm{~mm} \\
10^{\circ}\end{array}$ & & $-1,25 \mathrm{D}$ & & \\
\hline \multirow[t]{2}{*}{2} & $\begin{array}{l}\text { Ken. } \\
\text { Ima. }\end{array}$ & 20 & Schuler & $R$ & $\begin{array}{c}\text { uber } \\
12 \text { Tage }\end{array}$ & $\begin{array}{c}8,00 \mathrm{~mm} \\
180^{\circ}\end{array}$ & & $-0,5 \mathrm{D}$ & & $-1,25 \mathrm{D}$ \\
\hline & & & & I & $\begin{array}{c}\text { über } \\
12 \text { Tage }\end{array}$ & $\begin{array}{c}8,00 \mathrm{~mm} \\
180^{\circ}\end{array}$ & & $-0,75 \mathrm{D}$ & & $-0,5 \mathrm{D}$ \\
\hline \multirow[t]{2}{*}{3} & $\begin{array}{l}\text { Tok. } \\
\text { Sho. }\end{array}$ & 32 & $\begin{array}{l}\text { See- } \\
\text { offizier }\end{array}$ & $\mathrm{R}$ & 15 Jahre & $\mid \begin{array}{c}7,60 \mathrm{~mm} \\
175^{\circ}\end{array}$ & & $-1,0 \mathrm{D}$ & & $-1,25 \mathrm{D}$ \\
\hline & & & & $\mathrm{L}$ & $?$ & $\begin{array}{c}7,58 \mathrm{~mm} \\
180^{\circ}\end{array}$ & & $-0,75 \mathrm{D}$ & & $-1,0 \mathrm{D}$ \\
\hline \multirow[t]{2}{*}{4} & $\begin{array}{l}\text { To. } \\
\text { Ya. }\end{array}$ & 20 & Schüler & $\mathrm{R}$ & 2 Jahre & $\begin{array}{c}8,04 \mathrm{~mm} \\
180^{\circ}\end{array}$ & & $-0,75 \mathrm{D}$ & & $-1,00$ \\
\hline & & & & $\mathrm{L}$ & $?$ & $\mid \begin{array}{c}8,02 \text { max } \\
180^{\circ}\end{array}$ & & $-0,75 \mathrm{D}$ & & $-0,5 \mathrm{D}$ \\
\hline \multirow[t]{2}{*}{5} & $\begin{array}{l}\text { Sei. } \\
\text { Su. }\end{array}$ & 28 & $\begin{array}{l}\text { See- } \\
\quad \text { offizier }\end{array}$ & $\mathrm{R}$ & 45 Tage & $\begin{array}{c}8,28 \mathrm{~mm} \\
180^{\circ}\end{array}$ & $\begin{array}{c}8,30 \mathrm{~mm} \\
180^{\circ}\end{array}$ & $-0,5 \mathrm{D}$ & $-0,5 \mathrm{D}$ & $-0,75 \mathrm{D}$ \\
\hline & & & & $\mathrm{L}$ & & $\begin{array}{c}8,28 \mathrm{~mm} \\
180^{\circ}\end{array}$ & $\begin{array}{c}8,30 \mathrm{~mm} \\
180^{\circ}\end{array}$ & $-1,0 \mathrm{D}$ & $-1,0 \mathrm{D}$ & $-0,25 \mathrm{D}$ \\
\hline \multirow[t]{2}{*}{6} & $\begin{array}{l}\text { Toshi. } \\
\text { Nishi. }\end{array}$ & 22 & $\begin{array}{l}\text { See- } \\
\quad \text { offizier }\end{array}$ & $\mathrm{R}$ & 3 Jahre & $\begin{array}{c}7,79 \mathrm{~mm} \\
180^{\circ}\end{array}$ & & $-1,25 \mathrm{D}$ & & $-2,0 \mathrm{D}$ \\
\hline & & & & $\mathrm{L}$ & 3 Jahre & $\begin{array}{c}7,79 \mathrm{~mm} \\
10^{\circ}\end{array}$ & & $-1,20 \mathrm{D}$ & & $-2,0 \mathrm{D}$ \\
\hline \multirow[t]{2}{*}{7} & $\begin{array}{l}\text { Ji. } \\
\text { Ko. }\end{array}$ & 23 & $\begin{array}{l}\text { See- } \\
\quad \text { offizier }\end{array}$ & $\mathrm{R}$ & 240 Tage & $\begin{array}{c}7,90 \mathrm{~mm} \\
175^{3}\end{array}$ & $\begin{array}{c}7,90 \mathrm{~mm} \\
180^{\circ}\end{array}$ & $-0,5 \mathrm{D}$ & $-0,5 \mathrm{D}$ & $-1,0 \mathrm{D}$ \\
\hline & & & & $\mathrm{L}$ & $?$ & $\left|\begin{array}{c}7,88 \operatorname{mrn} \\
180^{\circ}\end{array}\right|$ & $\begin{array}{c}7,90 \mathrm{~mm} \\
180^{\circ}\end{array}$ & $-0,5 \mathrm{D}$ & $-0,75 \mathrm{D}$ & $-0,75 \mathrm{D}$ \\
\hline \multirow[t]{2}{*}{8} & $\begin{array}{l}\text { Ma. } \\
\text { Ogu. }\end{array}$ & 26 & $\begin{array}{l}\text { See- } \\
\text { offizier }\end{array}$ & $\mathrm{R}$ & $?$ & $\begin{array}{c}7,42 \mathrm{~mm} \\
8^{\circ}\end{array}$ & & $-0,75 \mathrm{D}$ & & 8 \\
\hline & & & & $L$ & $?$ & $\begin{array}{c}7,48 \mathrm{~mm} \\
6^{\circ}\end{array}$ & & $?$ & & $-0,75 \mathrm{D}$ \\
\hline
\end{tabular}


durch neue Behandlung mit Gummidruckverband.

Ubersichtliche Tabelle von Fällen 1-16.

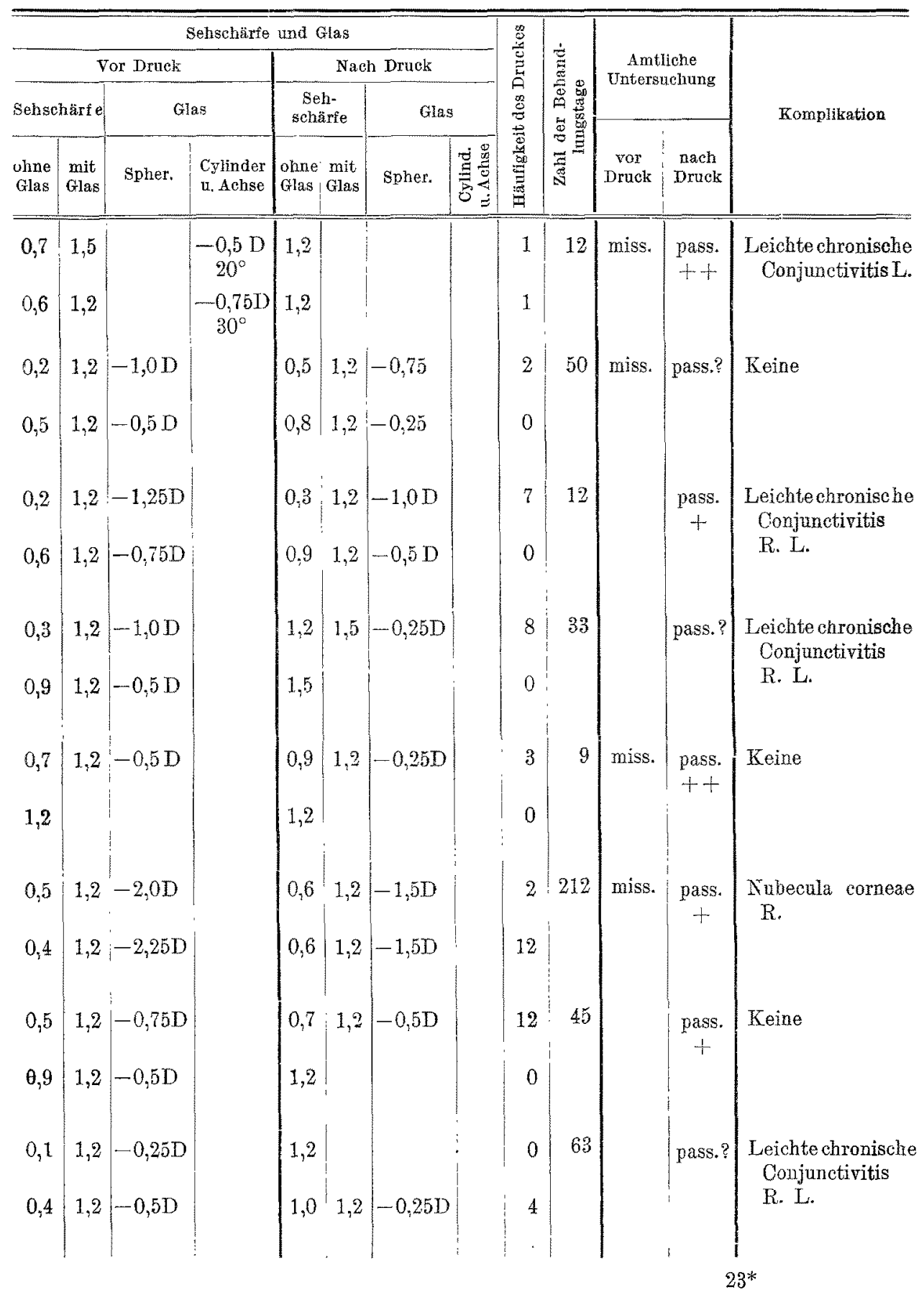


Tabelle II

\begin{tabular}{|c|c|c|c|c|c|c|c|c|c|c|}
\hline \multirow{3}{*}{$\begin{array}{l}\text { Fall } \\
\text { Nr. }\end{array}$} & \multirow{3}{*}{ Name } & \multirow{3}{*}{ Alter } & \multirow{3}{*}{ Beruf } & \multirow{3}{*}{$\begin{array}{c}\text { Recht } \\
R \\
\text { Links } \\
L\end{array}$} & \multirow{3}{*}{$\begin{array}{c}\text { Dauer der } \\
\text { Myopie } \\
\text { oder des } \\
\text { Astigm. }\end{array}$} & \multicolumn{4}{|c|}{ Javalsohes Ophthalmometer } & \multirow{3}{*}{ Skiaskop } \\
\hline & & & & & & \multicolumn{2}{|c|}{$\begin{array}{l}\text { schwächster Halb- } \\
\text { durchmesser der } \\
\text { Cornea und Achse }\end{array}$} & \multicolumn{2}{|c|}{$\begin{array}{l}\text { Differenz zwischen } \\
\text { sehwächstem und } \\
\text { stärkstem Meridian }\end{array}$} & \\
\hline & & & & & & $\begin{array}{l}\text { vor } \\
\text { Druek }\end{array}$ & $\begin{array}{l}\text { nach } \\
\text { Druek }\end{array}$ & $\begin{array}{l}\text { vor } \\
\text { Druek }\end{array}$ & $\begin{array}{c}\text { nach } \\
\text { Druck }\end{array}$ & \\
\hline \multirow[t]{2}{*}{9} & $\begin{array}{l}\text { Ryo. } \\
\text { Fuji. }\end{array}$ & 32 & $\begin{array}{l}\text { See- } \\
\text { offizier }\end{array}$ & $\mathrm{R}$ & $?$ & $\mid \begin{array}{c}8,01 \mathrm{~mm} \\
120^{\circ}\end{array}$ & $\begin{array}{c}8,01 \mathrm{~mm} \\
107^{\circ}\end{array}$ & $-1,25 \mathrm{D}$ & $-1,25 \mathrm{D}$ & $-1,25 \mathrm{D}$ \\
\hline & & & & L & $?$ & $\begin{array}{c}8,00 \mathrm{~mm} \\
95^{\circ}\end{array}$ & $\begin{array}{c}8,14 \mathrm{~mm} \\
100^{\circ}\end{array}$ & $-1,75 \mathrm{D}$ & $-1,5 \mathrm{D}$ & $-1,75 \mathrm{D}$ \\
\hline \multirow[t]{2}{*}{10} & $\begin{array}{l}\text { Ken. } \\
\text { Miya. }\end{array}$ & 27 & $\begin{array}{l}\text { See- } \\
\quad \text { offizier }\end{array}$ & $\mathrm{R}$ & 6 Jahre & $\begin{array}{c}7,80 \mathrm{~mm} \\
180^{\circ}\end{array}$ & & $-1,5 \mathrm{D}$ & & $-2,25 \mathrm{D}$ \\
\hline & & & & $\mathrm{L}$ & $\stackrel{?}{?}$ & $\mid \begin{array}{c}7,88 \mathrm{~mm} \\
176^{\circ}\end{array}$ & & $-1,5 \mathrm{D}$ & & $-2,0 \mathrm{D}$ \\
\hline \multirow[t]{2}{*}{11} & $\begin{array}{l}\text { Gon. } \\
\text { Naka. }\end{array}$ & 33 & $\begin{array}{l}\text { See- } \\
\text { offizier }\end{array}$ & $\mathrm{R}$ & 600 Tage & $\left(\begin{array}{c}7,81 \mathrm{~mm} \\
180^{\circ}\end{array}\right.$ & $\begin{array}{l}7,85 \mathrm{~mm} \\
180^{\circ}\end{array}$ & $-0,75 \mathrm{D}$ & $-0,75 \mathrm{D}$ & $\begin{array}{lll}-2,0 & \mathrm{D}\end{array}$ \\
\hline & & & & L & 600 Tage & $\mid \begin{array}{c}7,82 \mathrm{~mm} \\
170^{\circ}\end{array}$ & & $-1,25 \mathrm{D}$ & & $\begin{array}{lll}-1,5 & \mathrm{D}\end{array}$ \\
\hline \multirow[t]{2}{*}{12} & $\begin{array}{l}\text { Toku. } \\
\text { Uye. }\end{array}$ & 31 & $\begin{array}{l}\text { See- } \\
\quad \text { offizier }\end{array}$ & $\mathrm{R}$ & 11 Jahre & $172^{\circ}$ & $\left|\begin{array}{c}7,75 \mathrm{~mm} \\
167^{\circ}\end{array}\right|$ & $-0,75 \mathrm{D}$ & $-0,75 \mathrm{D}$ & $-1,25 \mathrm{D}$ \\
\hline & & & & $\mathrm{L}$ & 11 Jahre & $\frac{2 \operatorname{mm}}{160^{\circ}}$ & $\begin{array}{c}7,68 \mathrm{~mm} \\
162^{\circ}\end{array}$ & $-0,5 \mathrm{D}$ & $-0,25 \mathrm{D}$ & $-1,25 \mathrm{D}$ \\
\hline \multirow[t]{2}{*}{13} & $\begin{array}{l}\text { Shin. } \\
\text { Ama. }\end{array}$ & 32 & $\begin{array}{l}\text { See- } \\
\text { offizier }\end{array}$ & $\mathrm{R}$ & $\begin{array}{c}\text { uber } \\
30 \text { Tage }\end{array}$ & $\mid \begin{array}{c}75,3 \mathrm{~mm} \\
65^{\circ}\end{array}$ & $\mid \begin{array}{c}75,8 \mathrm{~mm} \\
85^{\circ}\end{array}$ & $-0,750$ & $-0,5 \mathrm{D}$ & \\
\hline & & & & $\mathrm{L}$ & $?$ & $\begin{array}{l}\text { ir* } \\
\text { regulär }\end{array}$ & $\begin{array}{c}74,6 \mathrm{~mm} \\
85^{\circ}\end{array}$ & irregulär & $-0, \tilde{0} \mathrm{D}$ & \\
\hline \multirow[t]{2}{*}{14} & $\begin{array}{l}\text { Ko. } \\
\text { Miya. }\end{array}$ & 25 & $\begin{array}{l}\text { See- } \\
\text { offizier }\end{array}$ & $\mathrm{R}$ & 2 Jahre & $\left(\begin{array}{c}75,5 \mathrm{~mm} \\
105^{\circ}\end{array}\right.$ & $\mid \begin{array}{c}76,1 \mathrm{~min} \\
145^{\circ}\end{array}$ & $-0,25 \mathrm{D}$ & $-0,75 \mathrm{D}$ & $-2,0 \mathrm{D}$ \\
\hline & & $:$ & & $\mathrm{L}$ & $?$ & $\begin{array}{c}75,4 \mathrm{~mm} \\
187^{\circ}\end{array}$ & $\begin{array}{c}75,8 \mathrm{~mm} \\
150^{\circ}\end{array}$ & $-0,25 \mathrm{D}$ & $-0,75 \mathrm{D}$ & $-1,5 \quad \mathrm{D}$ \\
\hline \multirow[t]{2}{*}{15} & $\begin{array}{l}\text { Tora. } \\
\text { Take. }\end{array}$ & 27 & $\begin{array}{l}\text { See- } \\
\quad \text { offizier }\end{array}$ & $\mathrm{R}$ & 8 Jahre & $\mid \begin{array}{c}7,40 \mathrm{~mm} \\
172^{\circ}\end{array}$ & $\left|\begin{array}{c}7,37 \mathrm{~mm} \\
175^{\circ}\end{array}\right|$ & $-1,0 \mathrm{D}$ & $-0,5 \mathrm{D}$ & $-2,0 \mathrm{D}$ \\
\hline & & ; & & L & $?$ & {$\left[\begin{array}{c}7,42 \mathrm{~mm} \\
12^{\circ}\end{array}\right.$} & $\left(\begin{array}{c}7,40 \mathrm{~mm} \\
16^{\circ}\end{array}\right.$ & $-1,25 \mathrm{D}$ & $-1,0 \mathrm{D}$ & $-2,0 \mathrm{D}$ \\
\hline \multirow[t]{2}{*}{16} & $\begin{array}{l}\text { Ku. } \\
\text { Kashi. }\end{array}$ & 29 & $\begin{array}{l}\text { See- } \\
\text { offizier }\end{array}$ & $\mathrm{R}$ & 6 Jahre & $\begin{array}{c}7,57 \mathrm{~mm} \\
163^{\circ}\end{array}$ & $\begin{array}{c}7,67 \mathrm{~mm} \\
170^{\circ}\end{array}$ & $-1,0 \mathrm{D}$ & $-1,0 \mathrm{D}$ & $-1,0 \mathrm{D}$ \\
\hline & & & & $\mathrm{L}$ & 6 Jahre & $\begin{array}{c}7,60 \mathrm{~mm} \\
140^{\circ}\end{array}$ & $\begin{array}{c}7,65 \mathrm{~mm} \\
180^{\circ}\end{array}$ & $-1,25 \mathrm{D}$ & $-0,75 \mathrm{D}$ & $-1.25 \mathrm{D}$ \\
\hline
\end{tabular}

*) Anmerkung: Amtliche Untersuchung pass ++ bedentet ,Passierung in die I. Klasse“; pass t bedeutet 
(Fortsetzung).

\begin{tabular}{|c|c|c|c|c|c|c|c|c|c|c|c|c|}
\hline \multicolumn{8}{|c|}{ Sehschärfe und Glas } & \multirow{4}{*}{ 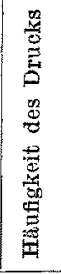 } & \multirow{4}{*}{ 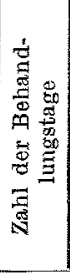 } & \multirow{3}{*}{\multicolumn{2}{|c|}{$\begin{array}{c}\text { Amtliche } \\
\text { Untersuchung }\end{array}$}} & \multirow{4}{*}{ Komplikation } \\
\hline \multicolumn{4}{|c|}{ Vor Druck } & \multicolumn{4}{|c|}{ Nach Druek } & & & & & \\
\hline \multicolumn{2}{|c|}{ Sehschärfe } & \multicolumn{2}{|c|}{ Glas } & \multicolumn{2}{|c|}{$\begin{array}{c}\text { Seh- } \\
\text { schärfe }\end{array}$} & \multicolumn{2}{|c|}{ Glas } & & & & & \\
\hline $\begin{array}{l}\text { ohne } \\
\text { Glas }\end{array}$ & $\begin{array}{l}\text { mit } \\
\text { Glas }\end{array}$ & Spher. & $\begin{array}{l}\text { Cylinder } \\
\text { in. Achse }\end{array}$ & $\begin{array}{l}\text { ohne } \\
\text { Glas }\end{array}$ & mit & Spher. & $\begin{array}{l}\text { Cylinder } \\
\text { u. Achse }\end{array}$ & & & $\begin{array}{c}\text { vor } \\
\text { Druck }\end{array}$ & $\begin{array}{l}\text { nach } \\
\text { Duuck }\end{array}$ & \\
\hline 0,7 & 1,2 & $-0,5 \mathrm{D}$ & & 1,2 & & & & 0 & & & & \\
\hline 0,1 & 1,2 & $-0,5 \mathrm{D}$ & $\begin{array}{c}-1,25 \mathrm{D} \\
90^{\circ}\end{array}$ & 0,8 & 1,2 & & $\begin{array}{c}-0,75 \mathrm{D} \\
90^{\circ}\end{array}$ & 2 & 15 & miss. & pass. ? & $\begin{array}{l}\text { Leichtes papil- } \\
\text { läres Trachom } \\
\text { R. L. }\end{array}$ \\
\hline 0,1 & 1,2 & $-2,25 \mathrm{D}$ & & 0,3 & 1,2 & $-1,5 \mathrm{D}$ & & 3 & 24 & & & Keine \\
\hline 0,2 & 1,2 & $-1,25 \mathrm{D}$ & & 0,4 & 1,2 & $-1,0 \mathrm{D}$ & & 0 & & & & \\
\hline 0,2 & 1,0 & $-1.0 \mathrm{D}$ & $\begin{array}{c}-1,0 \mathrm{D} \\
180^{\circ}\end{array}$ & 0,3 & 1,2 & $-1,0 \mathrm{D}$ & $\left|\begin{array}{c}-0,5 \mathrm{D} \\
180^{\circ}\end{array}\right|$ & 2 & 380 & miss. & & Akute Conjunc- \\
\hline 0,4 & 1,2 & $-0,75 \mathrm{D}$ & $\begin{array}{c}-1,0 \mathrm{D} \\
180^{\circ}\end{array}$ & 0,6 & $1,2 \mid$ & $-0,75 \mathrm{D}$ & $\mid \begin{array}{c}-0,75 \mathrm{D} \\
180^{\circ}\end{array}$ & 0 & & & & tivitis R.L. \\
\hline 0,5 & 1,2 & $-0,75 \mathrm{D}$ & & 0,9 & 1,2 & $-0,25 \mathrm{D}$ & & 4 & 56 & miss. & pass. & \\
\hline 0,3 & 1,2 & $-0,75 \mathrm{D}$ & & 0,9 & 1,2 & $-0,25 \mathrm{D}$ & & 5 & & & $t+$ & \\
\hline 0,3 & 1,2 & $-0,5 \mathrm{D}$ & $\begin{array}{c}-0,5 \mathrm{D} \\
90^{\circ}\end{array}$ & 0,7 & 0,9 & & $\begin{array}{c}-0,5 \mathrm{D} \\
90^{\circ}\end{array}$ & 4 & 60 & miss. & pass. & Leichtes Nar- \\
\hline 0,6 & 1,2 & $-0,5 \mathrm{D}$ & $\begin{array}{c}-0,5 \mathrm{D} \\
90^{\circ}\end{array}$ & 0,7 & 1,0 & & $\mid \begin{array}{c}-0,25 \mathrm{D} \\
90^{\circ}\end{array}$ & 0 & & & + & $\begin{array}{l}\text { bentrachom } \\
\text { R. L. }\end{array}$ \\
\hline 0,5 & 0,5 & & & 0,6 & 1,0 & $-0,75 \mathrm{D}$ & & 3 & 59 & miss. & pass. & Chronische \\
\hline 0,6 & 1,0 & $-0,75 \mathrm{D}$ & & 0,7 & 1,2 & $-0,75 \mathrm{D}$ & & 0 & & & + & $\begin{array}{l}\text { Conjunctivitis } \\
\text { R. L. }\end{array}$ \\
\hline 0,2 & 1,0 & $-1,5 \mathrm{D}$ & & 0,4 & 1,2 & $-1,0 \mathrm{D}$ & & 15 & 60 & miss. & pass. & Leichte \\
\hline 0,4 & 1,2 & $-1,25 \mathrm{D}$ & & 0,6 & 1,2 & $-1,0 \mathrm{D}$ & & 0 & & & + & $\begin{array}{l}\text { chronische } \\
\text { Conjunctivitis }\end{array}$ \\
\hline 0,5 & 1,2 & $-0,5 \mathrm{D}$ & $\begin{array}{c}-0,25 \mathrm{D} \\
180^{\circ}\end{array}$ & 0,8 & 1,2 & $-0,25 \mathrm{D}$ & & 3 & 63 & miss. & pass. & Leichtes \\
\hline 0,4 & 1,2 & $-0,75 \mathrm{D}$ & $\begin{array}{c}-0,25 \mathrm{D} \\
180^{\circ}\end{array}$ & 0,6 & 1,2 & $-0,5 \mathrm{D}$ & & 5 & & & + & $\begin{array}{l}\text { Trachom R. L. } \\
\text { Chaldzion L. }\end{array}$ \\
\hline
\end{tabular}

„Passiernng in die II. Klasse"; pass? bedeutet, wahrscheinllch passiert". I. Klasse ist die beste Klasse. 
Schon Laqueur (in Annal. d'ocul. 1869. t. LXI, p. 216) hat festgestellt, daß ein Druck, welcher von außen her auf den Bulbus ausgeübt wird, den Refraktionszustand merklich herabsetzt.

Weiss (1886) hat es an 2 Fällen nachgewiesen. Es waren Fälle von Myopie an Leuten von 21 und 18 Jahren, welche gelernt hatten, durch Anspannung der Lider in der Richtung nach außen mit dem Finger ihr Sehen bedeutend zu bessern. Der Krümmungsradius wurde in dem einen Falle von $6,939 \mathrm{~mm}$ auf $7,383 \mathrm{~mm}$, in dem anderen von $7,418 \mathrm{~mm}$ auf 8,575 vergrößert.

Darier (1899) hat eine günstige Wirkung der Druckmassage des Auges konstatiert. Das Akkomodationsvermögen wird erhöht, eine akkomodative Asthenopie prompt beseitigt. Der intraokulare Druck wird herabgesetzt, der Abfluß der Augenflüssigkeit offenbar durch Erweiterung des Hornhautiriswinkels vermehrt.

Domec (1912) hat beginnende Myopie durch rechtzeitig angewendetes und öfter wiederholtes Kneten des Auges verhütet oder wenigstens im Fortschreiten aufgehalten.

Dinoux (1912) verwendete Pilocarpin-Eserin, um beginnende Myopie zum Rückgang oder wenigstens zum Stillstand zu bringen. Ebenso wirkt nach ihm die Massage.

Alle bis jetzt angegebenen Methoden benutzen entweder einen einfachen Druckverband oder Massage. Meine Methode renwendet zum Unterschied besonders geformten Gummi, der bisher noch nicht gebraucht wurde. Die Massage wirkt durch Druck und Nachlaß kurze Zeit hintereinander; meine Methode ist nur Druck. Damit der Druck ohne plötzliche naturwidrige Schwankungen allmählich nachläßt, mit anderen Worten, damit der Druckeffekt ungefähr 9 stunden lang sich fortsetzt, habe ich für die Behandlung die Zeit vor dem Schlafengehen gewählt, nach Entfernung der Gummiform ist jede Massage und selbst das Berühren des Auges streng verboten.

$\mathrm{Zu}$ Tabelle 2 möchte ich einige Bemerkungen machen. Ich habe in dieser Tabelle getreulich alle Fälle, die ich mit Gummidruck gegen Myopie behandelt habe, aufgeführt mit 4 Ausnahmen. Bei diesen 4 Fällen bestanden Komplikationen mit Neuritis optica oder Conjunctivitis acuta oder in 2 Fällen mit Akkomodationskrämpfen. Bei jenen 4 Fällen habe ich nach der Behandlung meist gebesserte Sehschärfe gefunden, aber nie eine Verschlechterung.

Ich weiß, daß bei manchen Fällen von Myopie ein geringgradiger Akkommodationskrampf nebenherging, aber mit dem Akkommodationskrampf allein ist der ganze Heilvorgang nicht zu erklären.

Der Augenhintergrund war bei allen meinen Fällen intakt: nur bei Fall 4 und 6 auf beiden Augen Conus myopicus zu finden.

Die Skiaskopie sollte eigentlich vor und nach der Druckbehandlung 
gemacht $w \in$ rden, aber ich habe sie nicht regelmäßig ausgeführt, weil die skiaskopische Untersuchung bei einigen Fällen (Fall 9 und 12) so minimale Differenzen zeigte, daß eine objektive Besserung der Myopie auf Grund der Skiaskopie mit Sicherheit kaum behauptet werden kann. Skiaskopie wurde ohne Atropin in einer Entfernung von $50 \mathrm{~cm}$ vorgenommen. Wegen des dunkeln Hintergrundes ist die Skiaskopie des Japaners ohne Erweiterung der Pupille in einer größeren Entfernung schwierig.

Wenn man unmittelbar nach Fortnahme des Druckverbandes die Cornea mit dem Javalschen Ophthalmometer beobachtet, findet man eine beträchtliche Abflachung zusammen mit unregelmäßigem Astigmatismus.

Der unregelmäßige Astigmatismus verschwindet nach kurzer Zeit vollständig; von der Abflachung der Cornea scheint mir bisweilen etwas zurückzubleiben, wie zu ersehen aus Tabelle 2 unter ,schwächster Halbdurchmesser der Cornea", in Fällen 9, 11, 12, 16. Das Javal sche Ophthalmometer zeigte bei Fällen $9,12,13,15,16$ eine Verminderung des Astigmatismusgrades, wie aus Tabelle 2 hervorgeht. Ich bin der Meinung, daß beim Druckverband mit der einfachen konkaven Fläche, wie in Abb. 15 und 19, auch ein Astigmatismus korrigierender Effekt erzielt wird, weil der stärker gewölbte Meridian der Cornea mehr gedrückt wird als der schwächer gewölbte Meridian. Jedoch scheint mir für Astigmatismus die Gummiform mit zwei Vorstülpungen wie in Abb. 13 den Vorzug zu verdienen.

Druckverband mit einfacher konkaver Fläche gibt es in zwei Formen, Taggummiform wie in Abb. 19 und Nachtgummiform wie in Abb. 15 und 16. Taggummiform ist bei geschlossen behandeltem und geöffnetem nicht behandeltem Auge zu brauchen.

Beim Gebrauche der Taggummiform sitzt der Patient vor einem vertikal aufgehängten großen Spiegel, drei Meter von diesem entfernt: die Gesichtslinie richtet er nach seinem eigenen Gesicht, dessen Schatten er im Spiegel sieht. Er soll diesen Schatten nicht fest fixieren oder akkommodieren, sonst ermüdet er zu rasch, er soll zwar nicht viel blinzeln, doch ohne seine Aufmerksamkeit besonders auf diesen Punkt zu richten. Das gesunde Auge sei dabei nur halb offen, so daß die obere Hälfte der Cornea durch das Lid bedeckt ist.

Als zweckmäßige Vorübung empfiehlt sich vielleicht eine Art kontemplativer Ruhe, wie solche von der durch den Hindupriester Bodhidharma gegründeten, in Japan verbreiteten Zenshusekte gepflegt wird. Diese Übung besteht darin, in bestimmter Körperhaltung längere Zeit still zu sitzen, bei möglichster Abstraktion von allen Gedanken.

Im heißen Sommer ist die Gummidruckbehandlung möglichst zu vermeiden. Bindehautentzündungen müssen vorerst beseitigt sein. 
Um die Gummidruckmethode kennenzulernen, beginnt man zweckmäßigerweise mit der Staphylomabehandlung und geht erst später zur Myopie über, die Gummiformen (siehe Abb.6-9 vorhergehender Arbeit) sind sich ja einander sehr ähnlich, bei zentralem Staphylom sogar theoretisch völlig gleich. Die sog. Nachtgummiform wie in Abb. 15 ist zur Staphylombehandlung besser als eine Taggummiform wie in Abb. 19.

Eine Nachtgummiform zur Myopiebehandlung ist auf einem Auge bei geschlassenem Zustande der beiden Augen zu verwenden. Falls Nachtgummiform keine besondere Schmerzen verursacht, kann sie anstatt der Taggummiform auch bei sitzender Körperhaltung zur Myopiebehandlung gebraucht werden, während das nicht behandelte Auge geöffnet ist.

Der Verband ist ebenso anzulegen, wie in der vorhergehenden Arbeit beim Staphylom beschrieben (siehe Abb. 10); nur wird beim Staphylom zwischen Lid und Gummiform eine einfache Schicht Leinwand gelegt, bei der Myopie jedoch nicht. Keinesfalls darf die Gummiform oder Leinwand direkt mit der Cornea in Berührung kommen. Diesen Gummidruckverband darf man nicht wie sog. Druckverbände zu fest anlegen, sondern wie gewöhnliche Augenverbände.

Der Verband mit einer Nachtgummiform wird vor dem Sehlafengehen angelegt, schläft der Patient damit ein, so kann der Gummi ohne Schaden 4 bis 5 Stunden liegen bleiben, falls es nicht schmerzt. Bei Schmerz muß andererseits der Verband sogleich entfernt werden.

Die Dauer des Druckverbandes ist nach meinen Erfahrungen am zweckmäßigsten 30 Minuten bis zu 2 Stunden: die Norm ist eine Stunde.

Über die Dauer des Effekts kann ich noch nichts Sicheres sagen. Bei Seeoffizieren scheint der Erfolg lange anzuhalten, denn keiner von ihnen kam später mit einer Klage der Verschlechterung wieder zu mir, bisweilen empfahl einer dem anderen meine Behandlung. Von einzelnen habe ich erfahren, daß die Sehschärfe noch nach Jahren in gutem Zustande geblieben wäre.

Die Sehschärfeverbesserung als Folge meiner Behandlungsart kann man auf Tabelle I und II gut überblicken, z. B. von 0,1 auf 0,3 , von 0,3 auf $1,0 \mathrm{usw}$.

Auf den gleichen Tabellen ist auch vermerkt, daß sich die Stärke der zur Korrektion der Refraktionsfelder nötigen Gläser nach meiner Behandlung um $0,25 \mathrm{D}-0,75 \mathrm{D}$ verminderte.

Daraus geht hervor, daß sich meine Behandlungsart nur für Refraktionsanomalien geringerer Grade eignet und die Erfolge bei Myopie oder Astigmatismus von weniger ats ID dem Ideal nahekommen. Für solche Fälle möchte ich meine Gummidruckmethode den Fachkollegen zur Nachprüfung empfehlen. Denn die Möglichkeit, ohne Gläser eine 
Besserung der Sehschärfe zu erzielen, und zwar für die Dauer, bedeutet entschieden einen großen Gewinn, besonders für Männer, die sich einem Berufe widmen wollen, für den von Amts wegen hohe Anforderungen an die Sehsehärfe ohne Glas gestellt werden, wie beispielsweise in der Marine. Auch junge Damen suchen aus kosmetischen Gründen das Tragen von Gläsern zu vermeiden. Bei geringgradigen Anomalien läßt sich solchem Wunsche durch meine Methode vernünftigerweise Rechnung tragen.

\section{Literaturverzeichnis.}

Laqueur, Über die Hornhautkrümmung im normalen Zustande und unter pathologischen Verhältnissen. Arch. f. Ophthalmol. 30, 99. 1884. - Weiss, Ủber den. Einflaß der Spannung der Augenlider auf die Krümmung der Hornhaut und damit auf die Refraktion des Auges. Ber. d. 18. Vers. d. Ophthalmol. Ges. zu Heidelberg 1886, S. 72. - Darier, Die Augenmassage, Hornhautmassage und Vibrationsmassage. Die Druckmassage und ihre Wirkung auf die Akkommodation und Refraktion. Ophthalmol. Klinik 1889, Nr. 17. - Domec, Massage-pression, Myopie. Clin. ophtalmol. S. 474 et (Soc. franc d'ophtalmol. Congr. de mal,) Arch. d'ophtalmol. 32, 391. 1912. - Dianoux, Myopie et myotiques. Clin. ophtalmol. 1912, S. 68. - Inouye, Tatsuji, Über Astigmatismus durch Druck von Orbitaltumor. Nippon Ganka Gakkai Zashi 16, Nr. 16, S. 327. 1912. Nakamura, Bumpei, Über Krümmungsradius der Cornea. Ganka Rinsho Tho 8, 282. 1913. - Vogt, Durch Orbitaltumor bedingter Astigmatismus regularis der Cornea. Klin. Monatsbl. f. Augenheilk. 55, 652. 1915. - Inouye, Tatsuji, Vergleich zwischen Landoltscher ringförmiger und meiner hakenförmigen Sehprobentafel. Nip. Gan. Gak. Zas. 19, 399. 1915. - Inouye, Tatsuji, Neue Bezeichnung der Meridiane bei Astigmatismus. Nip. Gan. Gak. Zas. 19, 690. 1915. - Inouye, Tatsuj̈, Über Druckverband der Augen. (Gummidruck gegen Staphylom, Myopie und Entropium.) Nip. Gan. Gak. Zas. 21, 1153. 1917. - Satake, Shuichi, Experimentelle Hyperopie. Gan. Rin. Tho 13, 154. 1918. - Kusama, Kaname, Ein Fall von monokularer Diplopie durch Formveränderung der Cornea. Chuo Ganka Tho 10, 713. 1918. - Kavashima, Sei, Ein Fall von Hornhautastigmatismus durch Chalazion. Chuo Gan. Tho 10, 716. 1918. - Satake, Shuichi, Experimentelle Hyperopie. Gan. Rin. Iho 13, 154. 1918. 